\title{
BEITRÄGE ZUR GESCHICHTE DER SLAWISTIK
}



VERÖFFENTLICHUNGEN DES INSTITUTS FÜR SLAWISTIK HERAUSGEGEBEN VON H. H. BIELFELDT

NR. 30

\title{
BEITRÄGE
}

\section{ZUR GESCHICHTE DER SLAWISTIK}

\author{
Herausgeber \\ H. H. Bielfeldt und K. Horálek \\ Redaktion \\ H. Pohrt und M. Kudělka
}

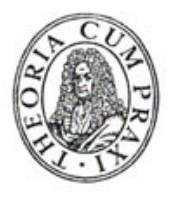

A K A D E M I E - V E R LA G B E R L I N

1964 
Erschienen im Akademie -Verlag GmbH, Berlin W 8, Leipziger StraBe 3-4

Copyright 1964 by Akademie -Verlag GmbH

Lizenz-Nr. 202 $\cdot 100 / 29 / 64$

Gesamtherstellung: Druckhaus „Maxim Gorki“, Altenburg

Bestellnummer : 2040/30 - ES 7 I 